\title{
Michael Kaiser, Harald Rosenbach (eds.), Max Weber in der Welt. Rezeption und Wirkung
} Tübingen, Mohr Siebeck, 2014, 243 p.

\section{Michael Löwy}

\section{(Q) OpenEdition}

\section{Journals}

Édition électronique

URL : http://journals.openedition.org/assr/27390

DOI : $10.4000 /$ assr. 27390

ISSN : $1777-5825$

\section{Éditeur}

Éditions de l'EHESS

Édition imprimée

Date de publication : 1 octobre 2015

Pagination : 321

ISBN : 978-2-7132-2515-4

ISSN : 0335-5985

\section{Référence électronique}

Michael Löwy, « Michael Kaiser, Harald Rosenbach (eds.), Max Weber in der Welt. Rezeption und

Wirkung ", Archives de sciences sociales des religions [En ligne], 172 I octobre-décembre, mis en ligne le 25 mai 2016, consulté le 24 septembre 2020. URL : http://journals.openedition.org/assr/27390 ; DOI https://doi.org/10.4000/assr.27390

Ce document a été généré automatiquement le 24 septembre 2020

(C) Archives de sciences sociales des religions 


\section{Michael Kaiser, Harald Rosenbach (eds.), Max Weber in der Welt. Rezeption und Wirkung}

Tübingen, Mohr Siebeck, 2014, 243 p.

Michael Löwy

\section{RÉFÉRENCE}

Michael Kaiser, Harald Rosenbach (eds.), Max Weber in der Welt. Rezeption und Wirkung, Tübingen, Mohr Siebeck, 2014, 243 p.

1 Ce volume réunit les communications présentées lors d'un Colloque international sur « Max Weber dans le monde », en juillet 2012 à l'université de Bonn. Comme dans tout recueil de ce genre, les travaux sont de valeur inégale, mais l'ensemble est tout de même intéressant. La plupart des textes concernent la réception de Weber dans différents pays, mais certains sont dédiés à des événements qui l'ont influencé, comme le séjour à Rome, le voyage aux États-Unis, ou la Première Guerre mondiale - ce qui introduit une certaine hétérogénéité dans le recueil. Les questions concernant les sciences sociales des religions sont présentes dans la plupart des communications, mais souvent de manière partielle et limitée.

Dans une brève introduction, les deux responsables pour l'édition du volume, Michael Kaiser et Harald Rosenbach, présentent le Colloque, parrainé par la Fondation des Instituts Allemands de Sciences Humaines (Geisteswissenschaften - concept intraduisible...) à l'Étranger. Un premier papier, par Edith Hanke, esquisse une vue générale de la réception internationale de Weber, les différentes traductions, etc. Ce sont des informations intéressantes, notamment sur la réception de Weber en Russie, en Chine et en Amérique Latine, même si on trouve quelques coquilles, par exemple, dans la transcription de noms brésiliens : le grand sociologue brésilien Sergio Buarque 
de Holanda est présenté comme "Sergio Baraque de Holanda ", et le journal "Diarios Associados » est désigné « Diaros Ascoiados ».

3 Bien entendu, il ne peut pas s'agir d'un bilan exhaustif, car beaucoup de pays sont absents du recueil, à commencer par... la France! Hinnerk Bruhns, dans son excellent article sur Weber et la Première Guerre mondiale, mentionne en passant que, pendant longtemps, le rapport de Weber à la France a été discuté, des deux côtés du Rhin, surtout du point de vue de son manque d'intérêt pour Durkheim. Les pays étudiés sont, dans l'ordre, le monde arabe en général, la Turquie, l'Égypte, la Russie, la Pologne, le Japon et les États-Unis.

Évidemment, la réception de Weber dans le monde arabe s'est beaucoup intéressée, comme le montre Stefan Leder, au rapport entre Islam et capitalisme. Les opinions à ce sujet sont loin d'être convergentes : tandis que Hamid Mahmud al-Aglan pense que la Charia est un obstacle au développement d'un capitalisme moderne, Barraq Zakhariya et Ridwan as-Sayyid sont persuadés qu'on peut trouver dans l'Islam des courants équivalents au calvinisme. On trouve un débat analogue en Turquie (article de Alexandre Toumarkine), où Sabri Ülgener, «le Weber Turc », pense que ce n'est pas, comme le prétendait Weber, le caractère guerrier de l'Islam qui a fait obstacle au capitalisme moderne dans l'Empire ottoman, mais l'absence d'une éthique du travail. Le débat en Égypte, analysé par Haggag Ali, a pris une autre tournure. Inspiré par le concept wébérien de sécularisation, Abdelwahab Elmessiri distingue entre deux types idéaux: le sécularisme partiel, qui sépare religion et politique, mais ne nie pas les valeurs morales et religieuses - et qui est donc compatible avec l'Islam - et le sécularisme total, propre à l'Occident, qui proclame une totale immanence, avec des conséquences souvent désastreuses.

5 Tandis que le chapitre sur la Russie (Dittmar Dahlmann) s'intéresse surtout aux écrits de Weber sur l'Empire des Tsars, où la religion prend peu de place, celui de Marta Buchholc sur la Pologne examine de près l'article de jeunesse (1892) de Weber sur les travailleurs saisonniers polonais en Allemagne de l'Est. Pour le sociologue, ces travailleurs représentent, à cause de leur religion catholique - imprégnée de magie et d'« enchantement » - un danger culturel pour l'Allemagne.

6 Étudiant la réception au Japon, Wolfgang Schwentker analyse d'abord les diverses traductions de l'Éthique Protestante $(1938,1955,1988)$ et les difficultés des traducteurs à trouver un vocabulaire japonais approprié. Curieusement, on trouve peu de références à cet ouvrage « classique » lors du Congrès Weber de Tokyo en 1999. Le grand débat sur le livre aura lieu au cours des années 2000, mais sous une forme assez particulière. En effet, les auteurs ne discutent pas de l'adéquation ou non des concepts wébériens à l'analyse des religions japonaises, mais des problèmes de traduction anglaise de l'Épître aux Corinthiens de Saint-Paul! Le sociologue de la culture Ha'nyu Tatsurô, dans un ouvrage pompeusement intitulé Le crime de Max Weber (2002), accuse l'auteur de l' Éthique Protestante d'avoir introduit à tort le terme anglais calling dans le débat sur l'éthique du travail protestante. Dans sa réponse polémique, le sociologue Orihara Hiroshi rappelle, avec bon sens, que ces détails ne mettent nullement en question la problématique du livre.

7 Nous arrivons enfin au texte qui nous semble de loin le plus intéressant du recueil, $d u$ point de vue des sciences sociales des religions. Il s'agit de l'essai de Peter Hersche sur les séjours de Max Weber à Rome (1901-1903) et son rapport au catholicisme - un thème rarement abordé par la littérature sur Weber. Son hypothèse principale - déjà 
suggérée par Hartmut Lehmann - est la suivante: frappé par la joie de vivre des catholiques italiens, et par leur riche architecture baroque, Weber s'est mieux rendu compte du caractère dur et ascétique du protestantisme calviniste, auquel il allait dédier son grand ouvrage de 1904. Peter Hersche va plus loin, et parle d'une véritable fascination de Weber pour le catholicisme italien, et d'un tournant dans sa vie suscité par l'agréable séjour romain, avec une prise de distance avec la rigueur protestante (y compris dans sa vie érotique). Il avance aussi l'hypothèse que l'Éthique Protestante devait être, à l'origine, une comparaison entre l'éthique catholique et la protestante. S'il ne l'a pas fait, c'est parce qu'il pensait manquer de compétence sur le catholicisme. Et par la suite, il ne s'est pas occupé de cette confession parce qu'il était pris par ses travaux sur les religions orientales.

8 L'explication est un peu courte... Pourquoi Max Weber s'est-il lancé dans l'étude du taoïsme et de l'hindouisme - qui lui ont sans doute posé des problèmes linguistiques et exigé des longues recherches sur un terrain culturellement étranger - plutôt que du catholicisme, religion de ses voisins de palier en Allemagne? Ce n'est sans doute pas par «manque de compétence ». L'absence du catholicisme dans l'œuvre de Weber reste encore à expliquer. Certes, il avait envers cet univers religieux une attitude ambigüe : enchantement lors du séjour romain, mais aussi une critique de la hiérarchie bureaucratique de l'Église - sa « Kaplanokratie » - et un rejet du catholicisme politique. Cependant, comme le montre Peter Hersche, il ne s'identifiait pas avec le Kulturkampf de l'État allemand protestant contre le catholicisme. Alors, comment rendre compte de ce silence assourdissant sur un millénaire de l'histoire de l'Occident, de la part de celui qui a fait de la recherche des origines de la rationalité occidentale l'objet central de son œuvre ? La question reste posée et mériterait une recherche.

Le dernier texte du recueil où il est question du religieux est celui de Lawrence A. Scaff sur la réception de Weber, et en particulier de l'Éthique Protestante, aux États-Unis. Comment expliquer l'extraordinaire succès de l'œuvre de Weber en Amérique? Un premier facteur serait le processus d'institutionnalisation universitaire de sa pensée, au cours de trois grandes "vagues » : les premières traductions et analyses, avec Talcott Parsons et Edward Shils, au cours des années 1930 ; l'arrivée de chercheurs allemands exilés dans les années 1930 et 1940 (Franz Neumann, Paul Lazarsfeld, Hans Gerth); la génération de l'après-guerre, notamment à la Columbia University (Robert Merton, C. Wright Mills). Ce qui nous semble manquer, dans cette partie du récit, c'est le rôle des grands penseurs de l'École de Francfort exilés aux USA, Theodor Adorno, Max Horkheimer ou Herbert Marcuse, certes critiques de Weber, mais fortement influencés par son œuvre.

10 Le deuxième facteur serait le séjour de Weber en Amérique (1904), qui lui a permis de mieux comprendre la nature de l'ascétisme protestant intra-mondain et son rapport au capitalisme. En étudiant - par observation participante - les sectes protestantes et la société américaine, il a découvert et développé dans l'Éthique Protestante trois récits qui ont capturé l'imagination de ses auditeurs et lecteurs américains : celui du volontarisme de la société nord-américaine, l'essor illimité d'associations religieuses ou profanes volontaires; celui du succès (achievement), personnifié par la figure de Benjamin Franklin, représentant parfait de l'esprit du capitalisme; et celui de la rédemption, sous forme de sécularisation de la quête du salut. Il y a donc eu une sorte d'identification culturelle entre l'auteur et ses lecteurs. 
11 L'hypothèse de Lawrence Scaff est intéressante, mais sa façon de présenter l'image de l'Amérique chez Weber est un peu trop idyllique. Heureusement, il donne lui-même des éléments pour nuancer le tableau. Il cite par exemple un passage de la correspondance qui révèle une distance critique évidente. Weber décrit ainsi le destin des communautés indigènes cherokee à Oklahoma, confrontées à la civilisation blanche : "dans sa folle course en avant, la culture capitaliste broie tout ce qui se trouve sur son chemin ». Quant à la présentation de Benjamin Franklin dans l'Éthique Protestante, comme le reconnaît Scaff, elle est bien plus ambivalente qu'une simple célébration du succès : la maîtrise (capitaliste) du monde ne conduit-elle pas à la cage d'acier de la domination? 